University of Nebraska - Lincoln

DigitalCommons@University of Nebraska - Lincoln

Controlled Covalent Functionalization of Multiwalled Carbon Nanotubes using [4 + 2] Cycloaddition of Benzocyclobutenes

Georgios Sakellariou

Haining Ji

Jimmy W. Mays

Nikos Hadjichristidis

Durairaj Baskaran

Follow this and additional works at: https://digitalcommons.unl.edu/usdoepub

Part of the Bioresource and Agricultural Engineering Commons

Sakellariou, Georgios; Ji, Haining; Mays, Jimmy W.; Hadjichristidis, Nikos; and Baskaran, Durairaj, "Controlled Covalent Functionalization of Multiwalled Carbon Nanotubes using [4 + 2] Cycloaddition of Benzocyclobutenes" (2007). US Department of Energy Publications. 63.

https://digitalcommons.unl.edu/usdoepub/63

This Article is brought to you for free and open access by the U.S. Department of Energy at DigitalCommons@University of Nebraska - Lincoln. It has been accepted for inclusion in US Department of Energy Publications by an authorized administrator of DigitalCommons@University of Nebraska - Lincoln. 


\section{Controlled Covalent Functionalization of Multiwalled Carbon Nanotubes using [4 + 2] Cycloaddition of Benzocyclobutenes}

Georgios Sakellariou, ${ }_{s}^{\dagger}$ Haining Ji, ${ }^{\dagger}$ Jimmy W. Mays, ${ }^{*},+, *$ Nikos Hadjichristidis, ${ }^{\S}$ and Durairaj Baskaran ${ }^{*, \perp}$

Polymer Science and Engineering Division, National Chemical Laboratory, Pune 411 008, India, Department of Chemistry, University of Athens, Athens, Greece, Department of Chemistry, University of Tennessee, Knoxville, Tennessee 37996, Chemical Sciences Division and Center for Nanophase Materials Sciences, Oak Ridge National Laboratory, Oak Ridge, Tennessee 37831

Received August 30, 2007

Revised Manuscript Received November 1, 2007

Surface modification of carbon nanotubes (CNTs) through covalent functionalization is vital for the development of high-performance composite materials, chemosensors, nanoelectronics, photovoltaic devices, as well as for a range of biomedical applications. ${ }^{1}$ Several methods have been developed to functionalize CNTs. ${ }^{2-8}$ The introduction of acid groups by acid digestion disrupts the structural integrity of CNTs. Apart from shortening the tubes, oxidatively generated acid groups are inhomogenously located at the tips of broken CNTs and, hence, functionalization using acid groups as precursors does not give a statistical distribution of functional groups throughout the surface of the CNTs.

The methods that have been explored for the functionalization of the $\mathrm{sp}^{2}$ network of the CNTs include addition reactions of azomethine ylides, ${ }^{3}$ bromomalonates, ${ }^{4}$ diazonium salts, ${ }^{5}$ nucleophilic carbenes, ${ }^{6}$ nitrenes, ${ }^{7}$ and free radicals. ${ }^{8}$ These methods can produce a higher extent of functionalization compared to the oxidative method. Recently, the Diels-Alder reaction has been used for the functionalization of single-wall carbon nanotubes, ${ }^{9-11}$ requiring the assistance

* Corresponding author. E-mail: baskaran@utk.edu (D.B.); jimmymays@utk.edu (J.W.).

† University of Tennessee.

Oak Ridge National Laboratory.

${ }^{\S}$ University of Athens.

${ }^{\perp}$ National Chemical Laboratory.

(1) Tasis, D.; Tagmatarchis, N.; Bianco, A.; Prato, M. Chem. Rev. 2006, 106, 1105-1136.

(2) Baskaran, D. In Encyclopedia of Inorganic Chemistry, 2nd ed.; Bruce King, R., Ed.; John Wiley \& Sons: Chichester, U.K., 2005; Vol. II, pp 730-764.

(3) Georgakilas, V.; Kordatos, K.; Prato, M.; Guldi, D. M.; Holzinger, M.; Hirsch, A. J. Am. Chem. Soc. 2002, 124, 2002.

(4) Coleman, K. S.; Bailey, S. R.; Fogden, S.; Green, M. L. H. J. Am. Chem. Soc. 2003, 125, 8722-8723.

(5) Bahr, J. L.; Yang, J.; Kosynkin, D. V.; Bronikowski, M. J.; Smalley, R. E.; Tour, J. M. J. Am. Chem. Soc. 2001, 123, 6536-6542.

(6) Holzinger, M.; Vostrowsky, O.; Hirsch, A.; Hennrich, F.; Kappes, M.; Weiss, R.; Jellen, F. Angew. Chem., Int. Ed. 2001, 40, 4002.

(7) Holzinger, M.; Abraham, J.; Whelan, P.; Graupner, R.; Ley, L.; Hennrich, F.; Kappes, M.; Hirsch, A. J. Am. Chem. Soc. 2003, 125, 8566-8580.

(8) Umek, P.; Seo, J. W.; Hernadi, K.; Mrzel, A.; Pechy, P.; Mihailovic, D. D.; Forro, L. Chem. Mater. 2003, 15, 4751-4755.

(9) (a) Lu, X.; Tian, F.; WAng, N.; Zhang, Q. Org. Lett. 2002, 4, 43134315. (b) Zhang, L.; Yang, J.; Edwards, C. L.; Alemany, L. B.; Khabashesku, V. N.; Barron, A. R. Chem. Commun. 2005, 3265.
Scheme 1. Diels-Alder Adduct of Various Benzocyclobutenes with Multiwalled Carbon Nanotubes

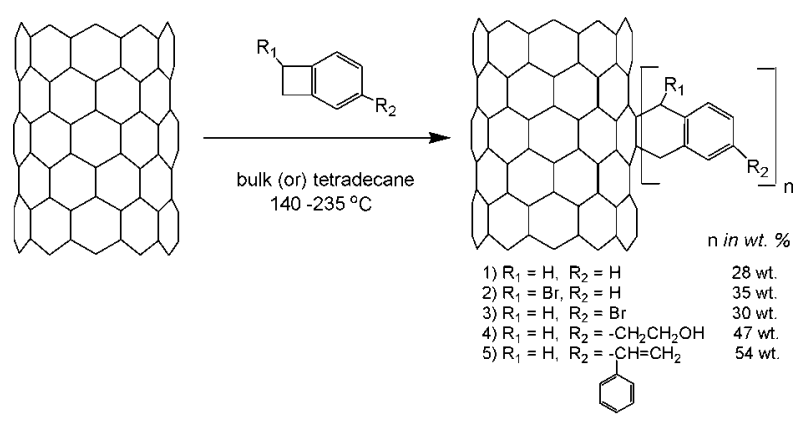

of microwave irradiation for the functionalization of soluble SWNTs,${ }^{10}$ and in another case, the reaction was performed in the presence of transition metal catalyst under highpressure. ${ }^{11}$ Nevertheless, these reactions do not exhibit control over the degree of functionalization and require removal of the catalyst ${ }^{11}$ and the dissolution of CNTs. ${ }^{10}$

Benzocyclobutene is a class of diene which can be used in the Diels-Alder reaction with the $\mathrm{sp}^{2}$ carbons of aromatic system through an $o$-quinodimethane intermediate. ${ }^{12}$ The reaction of benzocyclobutene with CNTs has not been performed prior to this work, although its feasibility has been shown theoretically. ${ }^{9}$ Here, we report a controlled functionalization of multiwalled carbon nanotubes through Diels-Alder [4 + 2] cycloaddition of various substituted benzocyclobutenes and investigate the extent of control over the functionalization, in bulk and in solution at different temperatures. The stained ring of the benzocyclobutene is susceptible to thermal ring opening to form o-quinodimethane which can undergo [4 4 2] DielsAlder cycloaddition exclusively to $6-6$ bonds of fused aromatic rings. ${ }^{12}$ Accordingly, in an attempt to add organic moieties on the surface of CNTs, several substituted benzocyclobutenes $(\mathbf{1}-\mathbf{5})$ were reacted with multiwalled carbon nanotubes (MWNTs; Scheme 1). Benzocyclobutenes containing a hydroxyl group (4, BCB- $\left.\mathrm{CH}_{2} \mathrm{CH}_{2} \mathrm{OH}\right)$ and 1-phenyl-1-benzocyclobutene ethylene (5, BCB-DPE) were synthesized from the reaction of a Grignard reagent of 4-bromobenzocyclobutene with ethylene oxide and acetophenone, respectively. Experiments were performed in bulk as well as in tetradecane. In a typical experiment, $20 \mathrm{mg}$ of pristine MWNTs were reacted in bulk with $150 \mathrm{mg}$ of benzocyclobutene (BCB) at different temperatures ranging from 140 to $235^{\circ} \mathrm{C}$ for $24 \mathrm{~h}$. In case of a reaction in tetradecane, $100 \mathrm{mg}$ of MWNTs was taken in $5 \mathrm{~mL}$ of tetradecane and 1 $\mathrm{mL}$ of $\mathrm{BCB}$ or substituted $\mathrm{BCB}$ was added dropwise via a syringe pump with vigorous stirring under argon.

After the reaction, the MWNTs were collected by washing with copious amount of tetrahydrofuran (THF) and filtered using a Teflon membrane $(0.2 \mu \mathrm{m}$ pore). The washing was

(10) Delgado, J. L.; Cruz, P.; Langa, F.; Urbina, A.; Casado, J.; Navarrete, J. T. L Chem. Commun. 2004, 1734-1735.

(11) Medard-Moyon, C.; Dumas, F.; Doris, E.; Mioskowski, C. J. Am. Chem. Soc. 2006, 128, 14764.

(12) Segura, J. L.; Martin, N. Chem. Rev. 1999, 99, 3199-3246. 

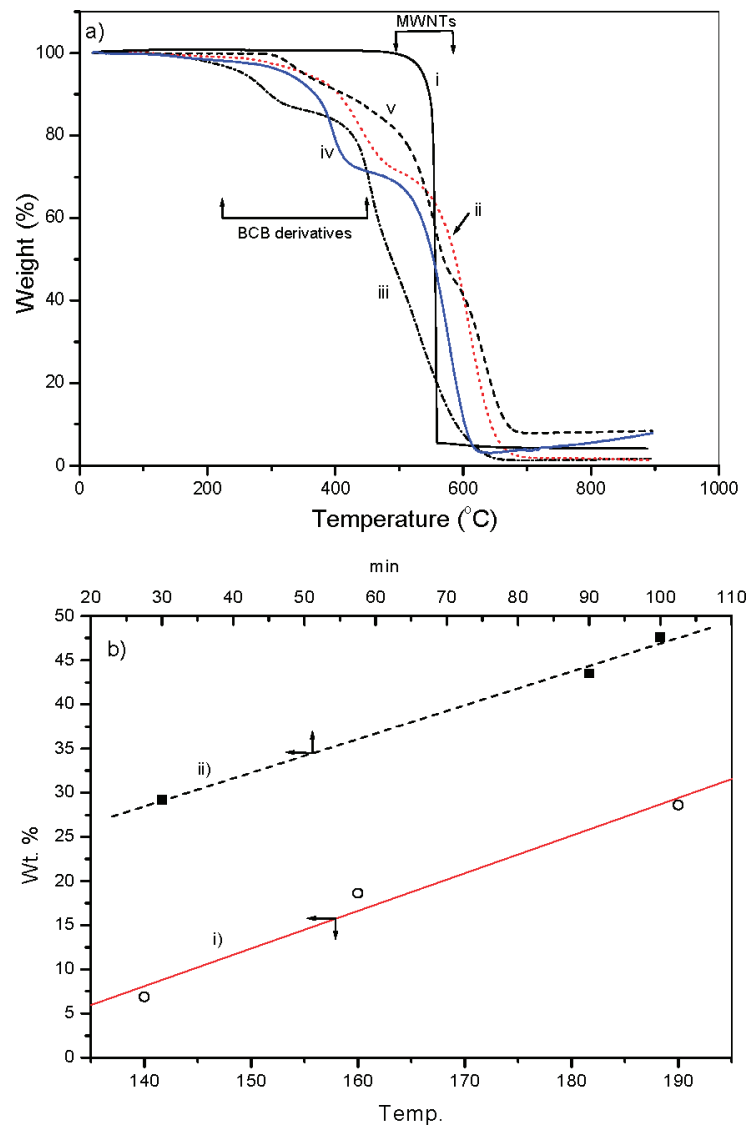

Figure 1. (a) TGA of MWNTs grafted with various derivatives of BCB: (i) pristine MWNTs, (ii) MWNTs- $g$-(BCB) ${ }_{n}, 28$ wt \% BCB, (iii) MWNTs$g$-(BCB-Br $)_{n}, 35$ wt $\%$ BCB-Br, (iv) MWNTs- $g$ - $\left(\mathrm{BCB}-\mathrm{CH}_{2} \mathrm{CH}_{2} \mathrm{OH}\right)_{n}, 47$ wt $\%$ BCB- $\mathrm{CH}_{2} \mathrm{CH}_{2} \mathrm{OH}$, and v) MWNTs- $g$-(BCB-DPE) ${ }_{n}, 54$ wt $\%$ BCBDEP. (b) Extent of functionalization depending on the reaction temperature and duration: (i) bulk reaction of $\mathrm{BCB}$ with MWNTs at different temperature for $24 \mathrm{~h}$, and (ii) dropwise addition of $\mathrm{BCB}-\mathrm{CH}_{2} \mathrm{CH}_{2} \mathrm{OH}$ at $235^{\circ} \mathrm{C}$ for different duration

repeated several times until the filtrate showed the absence of BCB by thin-layer chromatography. Benzocyclobutenes containing hydroxyl (4) and 1-phenyl-1-benzocyclobutene ethylene (5) were reacted in tetradecane using dropwise addition. The side-wall-functionalized MWNTs (MWNTs$g-(\mathrm{BCB})_{n}$ ) each having different substitution in BCB, $\mathbf{1 - 5}$, were dried at $60^{\circ} \mathrm{C}$ for $5 \mathrm{~h}$ under a vacuum and characterized using FT-IR, UV-vis absorption spectroscopy, Raman spectroscopy, and thermogravimetric analysis (TGA).

The quantity of BCBs attached to the surface of MWNTs$g$-(BCB $)_{n}$ was determined from TGA, which showed two distinct weight losses: (i) starting from 200 to $480{ }^{\circ} \mathrm{C}$ and (ii) a major weight loss at $590-625{ }^{\circ} \mathrm{C}$. The decompositions in the temperature ranges $200-480{ }^{\circ} \mathrm{C}$ and $590-625^{\circ} \mathrm{C}$ were assigned to the attached BCBs and the MWNTs, respectively (Figure 1a). A different decomposition pattern was observed for 1-5 related to the nature of the substituted BCB. The TGA performed under $\mathrm{N}_{2}$ showed presence of two steps of decomposition for the grafted $\mathrm{BCB}$ derivatives in the temperature range $200-480^{\circ} \mathrm{C}$. The amount of BCBs grafted to the tubes varies from 6 to $54 \mathrm{wt} \%$ depending on the reaction temperature as well as the mode of addition (Figure 1b).

As the reaction proceeds through the formation of $o$ quinodimethane radicals, the rate of Diels-Alder adduct formation is dependent on the temperature of the reaction. The control of the process is evident through an increase in weight percent of BCB on the surface of MWNTs with increasing reaction temperature in bulk (Figure $1 \mathrm{~b}-\mathrm{i}$ ). At $140{ }^{\circ} \mathrm{C}$, only $6.9 \mathrm{wt} \%$ BCB was grafted on the surface of the MWNTs. On the other hand, when the reaction was performed at $190{ }^{\circ} \mathrm{C}$, the grafting of $\mathrm{BCB}$ increased up to $28.6 \mathrm{wt} \%$. The reaction of 1-bromobenzocyclobutene (2) with MWNTs in bulk gave MWNTs$g$-(BCB-Br) ${ }_{n}$ with 35 wt \% functionalization (Supporting Information). Similarly, another isomeric bromobenzocyclobutene (3) also gave $30 \mathrm{wt} \%$ functionalization determined by TGA. However, it was observed that a prolonged reaction time has no significant influence on the weight percent of grafting, which could be attributed to the dimerization of $o$-quinodimethane radical intermediate. For example, reaction of $\mathbf{1}$ and $\mathbf{3}$ with MWNTs performed in bulk for 8 and $24 \mathrm{~h}$ produced a similar weight percent functionalization. To minimize the dimerization of BCBs, we kept the substituted BCBs, 4 and 5, at room temperature and added them dropwise into tetradecane-containing MWNTs at $235^{\circ} \mathrm{C}$.

The dropwise addition method significantly improves the percentage of grafting depending on the duration of the reaction. The reaction performed with $\mathbf{4}$ was kept stirring for 30,90 , and $100 \mathrm{~min}$, and the TGA revealed that the weight percent decomposition of the substituted BCB increased from $29.2,43.5$, and $47.6 \mathrm{wt} \%$, respectively. This indicates that the Diels-Alder reaction using BCB is controllable and the extent of grafting on to the surface of the MWNTs can be tailored using a suitable reaction temperature and time (Figure $1 \mathrm{~b}-\mathrm{ii}$ ).

The FT-IR spectrum of the various substituted MWNTs$g$-(BCB $)_{n}$ reveals characteristic vibrations of aromatic and $\mathrm{CH}$ modes ( $\sim 3010$ and $2930 \mathrm{~cm}^{-1}$, respectively) along with the characteristic vibration modes for the substitution group, confirming the presence of substituted BCB on the MWNTs (Supporting Information). The UV-vis absorption spectrum of 1 (Figure $2 \mathrm{a}-\mathrm{i}$ ) shows a broad absorption signal monotonously decreasing from 250 to $900 \mathrm{~nm}$ corresponding to the MWNTs. Similarly, MWNTs- $g-(\mathrm{BCB}-\mathrm{Br})_{n}(\mathbf{2})$ also confirms the presence of MWNTs (Figure 2a-ii). The Raman spectra of these functionalized MWNTs are similar to the pristine MWNTs showing a characteristic tangential band at $\sim 1566$ $\mathrm{cm}^{-1}$ and a disorder band at $\sim 1340 \mathrm{~cm}^{-1}$. However, the ratio of these bands $\left(I_{\mathrm{d}} / I_{\mathrm{t}}\right)$ is slightly high in the case of functionalized samples as compared to pristine MWNTs, indicating the introduction of $\mathrm{sp}^{3}$ carbons on the surface of the MWNTs (Figure 2b).

Covalent functionalization of MWNTs with polymer using a grafting-to strategy is known in the literature. ${ }^{2}$ It was expected that a polymer containing benzocyclobutene repeat units such as poly(4-vinyl benzocyclobutene) (PVBCB) would react at multiple sites on the surface of the MWNTs. If the proximity of the tubes permits, interlinking of MWNTs can be realized. The PVBCB was synthesized as reported elsewhere using anionic polymerization of 4-vinyl benzocyclobutene and reacted with MWNTs. ${ }^{13}$ Accordingly, 20 $\mathrm{mg}$ of MWNTs was refluxed in $15 \mathrm{~mL}$ of diphenylether containing $20 \mathrm{mg}$ of PVBCB $\left(M_{\mathrm{n}, \mathrm{SEC}}=30000 \mathrm{~g} / \mathrm{mol}, M_{\mathrm{w}} /\right.$

(13) Sakellariou, G.; Baskaran, D.; Hadjichristidis, N.; Mays, J. W. Macromolecules 2006, 39, 3522-3530. 

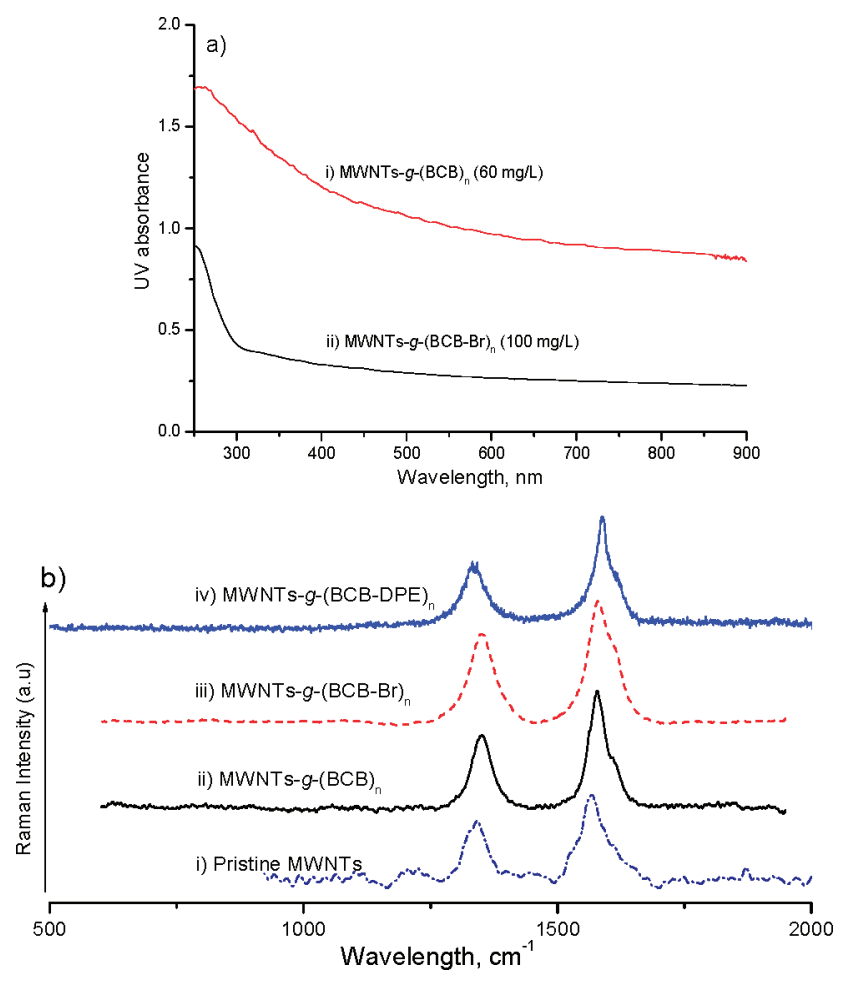

Figure 2. (a) UV-vis absorbance of benzocyclobutene-grafted MWNTs solutions in $\mathrm{CHCl}_{3}$ : (i) MWNTs- $g$-(BCB) $)_{n}(60 \mathrm{mg} / \mathrm{L}, n=28 \mathrm{wt} \%)$, and (ii) MWNTs- $g$-(BCB-Br $)_{n}(100 \mathrm{mg} / \mathrm{L}, n=35 \mathrm{wt} \%)$. (b) Raman spectra of various benzocyclobutene grafted MWNTs.

$\left.M_{\mathrm{n}}=1.05\right)$ at $240{ }^{\circ} \mathrm{C}$ for $2 \mathrm{~h}$. After the reaction, the MWNTs- $g$-(PVBCB $)_{n}$ were washed with copious amount of tetrahydrofuran and recovered by filtration and dried at 90 ${ }^{\circ} \mathrm{C}$ for $4 \mathrm{~h}$. The recovered MWNT- $g$-(PVBCB $)_{n}$ had a weight increase of $19 \mathrm{mg}$ ( $95 \mathrm{wt} \%$ ), indicating the total amount of PVBCB had undergone cross-linking via inter- or intramolecular reactions. The TGA of MWNTs- $g$-(PVBCB $)_{n}$ showed a $4 \%$ weight increase at $260{ }^{\circ} \mathrm{C}$ and $50 \%$ weight loss up to $500{ }^{\circ} \mathrm{C}$ corresponding to the grafted PVBCB on the MWNTs. The decomposition of MWNTs is clearly seen at $568{ }^{\circ} \mathrm{C}$. The weight increase is attributed to the reaction of residual pendant $\mathrm{BCB}$ groups of the polymer with oxygen present in the furnace. This confirms that the $\mathrm{BCB}$ groups present in the polymer have partially undergone the grafting reaction with MWNTs (Supporting Information). It is also known that PVBCB undergoes intramolecular cross-linking to form organic nanoparticles. ${ }^{14}$ The MWNTs- $g$-(PVBCB $)_{n}$ obtained was found soluble in THF and $\mathrm{CHCl}_{3}$ at low concentration (Supporting Information).

The TEM analysis of a sample prepared by drop-coating a solution of MWNTs- $g$-(PVBCB $)_{n}$ on a carbon grid showed the presence of cross-linked organic polymer as nanoclusters on the surface of MWNTs (Figure 3). The nanoclusters on the surface of MWNTs are nonuniformly located on the surface, which could be attributed to a random heterogeneous Diels-Alder reaction of benzocyclobutene pendants of polymer with $\mathrm{sp}^{2}$ carbons of the MWNTs. It appears that once the chemical anchoring of polymer occurs at $\mathrm{sp}^{2}$ carbon of the MWNTs, the

(14) Harth, E.; Horn, B. H.; Lee, V. Y.; Germack, D. S.; Gonzales, C. P.; Miller, R. D.; Hawker, C. J. J. Am. Chem. Soc. 2002, 124, 86538660.
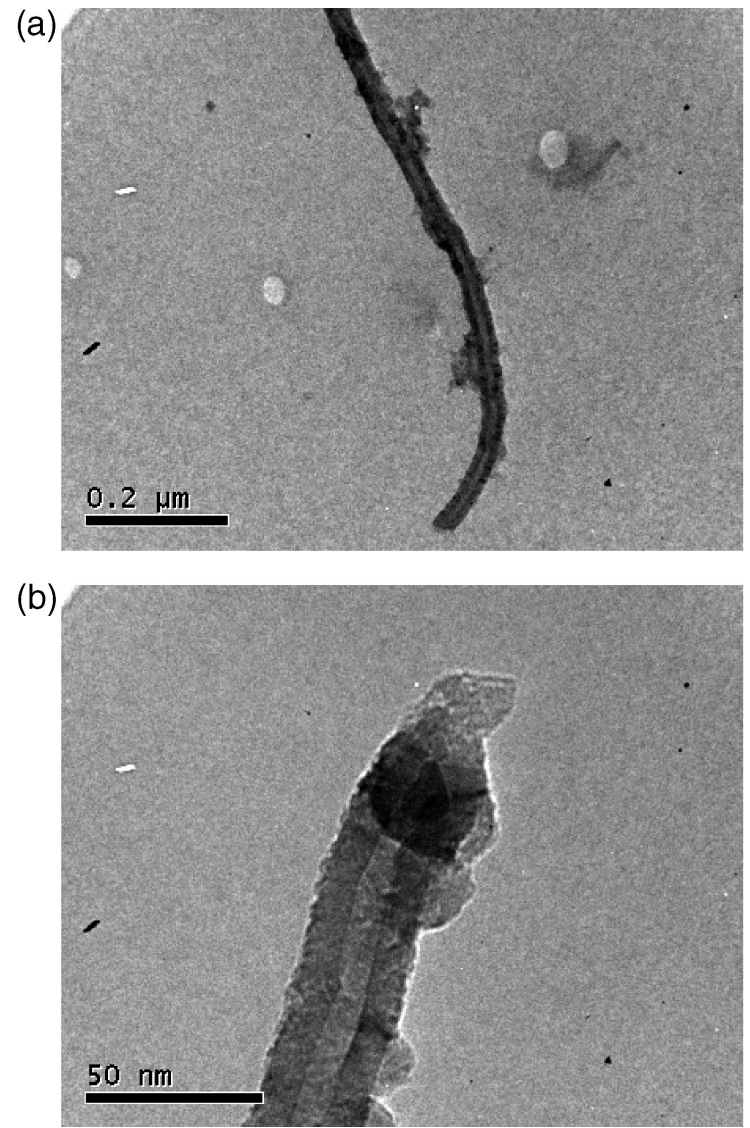

Figure 3. (a, b) Organic nanoclusters of cross-linked poly(4-vinylbenzocyclobutene) on the surface of MWNTs.

remaining tangling polymer chain undergoes inter- and intramolecular cross-linking reaction to form a collapsed polymeric nanocluster on the surface of the MWNTs. The size of nanocluster ranging from 5 to $13 \mathrm{~nm}$ also suggests that it involves reactions of intermolecular PVBCB on the surface of MWNTs. It is possible that by controlling the PVBCB chainlength or molecular weight, one can also control the size of added globs on the surface of MWNTs. The formation of polymeric nanoclusters on the surface of MWNTs through covalent cross-linking can be used to connect MWNTs to surfaces suseptible to radical intermediate.

In conclusion, we report a simple Diels-Alder reaction for the functionalization of MWNTs using benzocyclobutene. The extent of functionalization can be controlled by using the appropriate temperature and mode of addition of the reagent and by controlling the reaction duration. The use of polymer containing pendant benzocyclobutene produces cross-linked organic nanoclusters covalently connected to the surface of the MWNTs.

Acknowledgment. We acknowledge U.S. Department of Energy (DE-AC05-00OR22725) and NCL-CSIR, India (MLP 007926), E.U.-European Social Fund and the Greek Ministry of Development, for financial support. DB thanks Center for Material Characterization, NCL, India for TEM analysis.

Supporting Information Available: Experimental section and characterization details such as TGA of $\mathbf{1}$ and 2, FT-IR of $\mathbf{4}$ and $\mathbf{5}$, and more TEM (PDF). This material is available free of charge via the Internet at http://pubs.acs.org.

CM702470X 